\title{
Janus kinase inhibitors and fields of usage
}

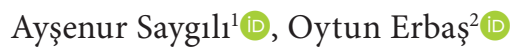 \\ ${ }^{1}$ Inönü University Molecular Biology and Genetics Graduate, Malatya, Turkey \\ ${ }^{2}$ Department of Physiology, Demiroğlu Bilim University Faculty of Medicine, Istanbul, Turkey
}

\begin{abstract}
Jak regions have been identified on chromosome 1, 9, and 19. Cytokines and receptors in the janus kinase/signal transducers and activators of transcription pathway are involved in cell signaling. In order to treat damages in the Jak pathway, molecules called inhibitors have been studied and used for treatment.

Keywords: Inhibitor, janus kinase, chromosome, signal path.
\end{abstract}

\section{JANUS KINASE (JAK) MECHANISM}

The differentiation, growth, and longevity of hematopoietic cells are controlled by a group of growth factors known as cytokines. Cytokines bind to their receptors, resulting in the activation of Janus Kinase receptor-related tyrosine kinases and thus intracellular signal transmission. ${ }^{[1,2]}$

The JAK family is the name given to a group of tyrosine kinases (without intracellular receptors) that play a role in cytokine-mediated signal transduction. This is known as the JAKSTAT (Signal Transducers and Activators of Transcription) pathway. Transcription factors in this pathway are called STATs. The JAKSTAT pathway is crucial in hematopoiesis as it regulates hematopoietic cells in terms of division, differentiation, and apoptosis. It also plays a role in embryonic development and inflammation..$^{[3,4]}$

The JAK family consists of four members: JAK1, JAK2, JAK3, and Tyrosine Kinase 2 (TYK2). These proteins do not work alone. In 1989, it was established that amino acid sequences in the catalytic regions of all known tyrosine kinases of hematopoietic cell lines were similar and identified as such. ${ }^{[5,6]}$

JAK family members have different chromosomal locations. JAK1 is found at 1p31.3, JAK2 at 9p24; JAK3 at 19p13.1, and TYK at 19p13.1 (Figures 1, 2). ${ }^{[7,8]}$ As JAKs are large proteins, they contain over a thousand amino acids. There are seven different JAK homology regions that make up the structural portion of JAK members. ${ }^{[9,10]}$ While JAK1, JAK2, and TYK2 are expressed by almost every cell in mammals, JAK3 is primarily expressed in hematopoietic and lymphoid tissues. ${ }^{[11]}$ Mutation of the JAK2 gene, which encodes tyrosine kinase, a protein involved in the JAK/STAT signaling pathway, was identified as the guanine nucleotide of the $14^{\text {th }}$ exon and $1849^{\text {th }}$ position changed with thymine (G1849T), leading to the V617F mutation, in which phenylalanine is coded instead of valine at the $617^{\text {th }}$ position in the synthesized protein..$^{[12-14]}$

JAK3 is the only JAK kinase that binds to the common signal transduction subunit $\gamma_{c}$ (gamma) of the receptor complex of several cytokines (IL2, IL4, IL7, IL9, IL15, and IL21). Hereditary 
(a)

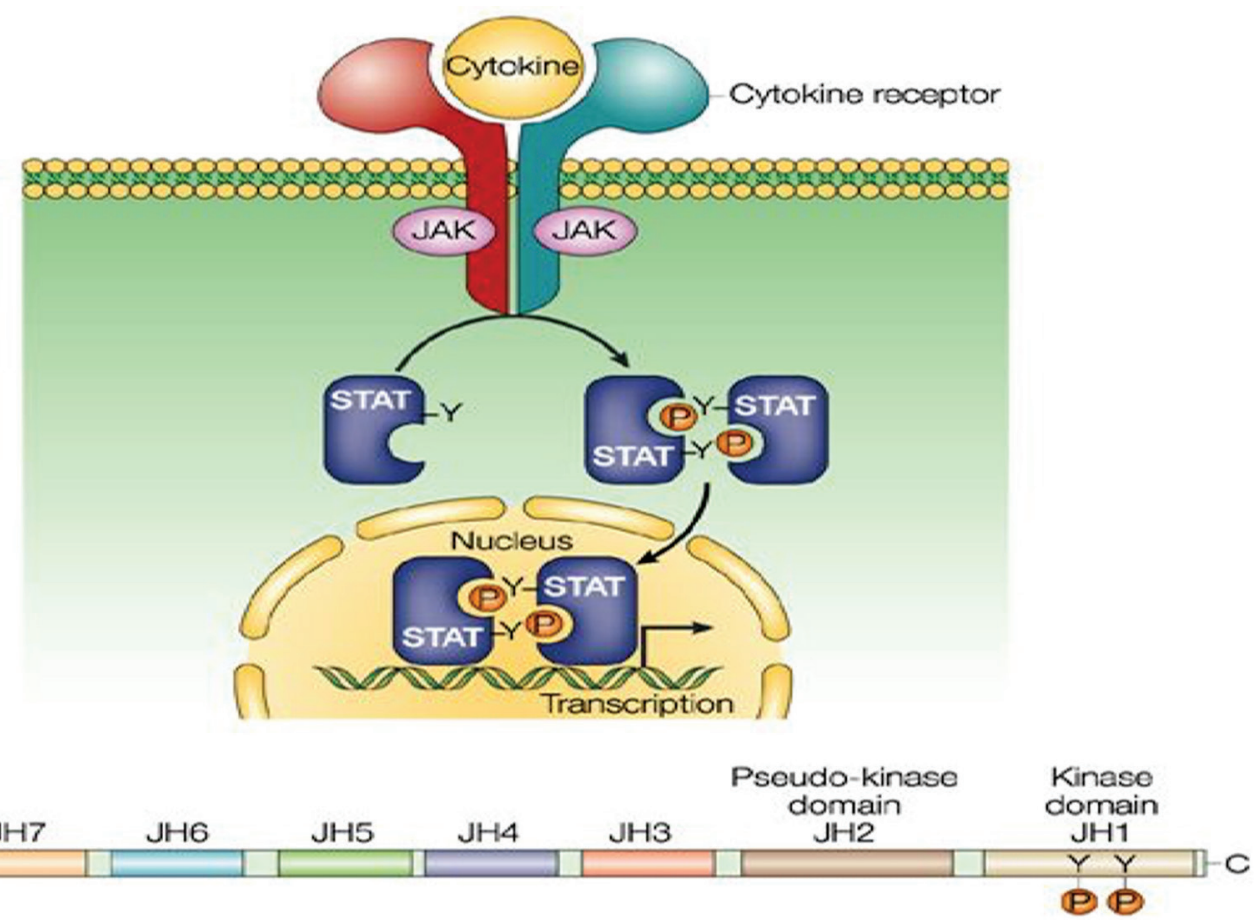

Figure 1. (a) JAK-STAT Pathway. (b) Regions on the JAK gene..$^{[7]}$

JAK3 and $\gamma c$ mutations lead to severe combined immunodeficiency (SCID). Patients with TYK2 function loss also develop immune deficiency. Therefore, JAK3 and TYK2 inhibition requires the most caution when using JAK inhibitors. ${ }^{[15]}$

Janus kinases regulate signal production of cytokine receptors. Janus kinases enzymes that bind to cytokine receptors on the cell surface, upon binding to the cytokine receptor gland, become phosphorylated and the receptor undergoes dimerization. When JAK is phosphorylated, it becomes activated. Activated JAK-receptor complex becomes phosphorylated and activates STATs which are substrate molecules and transcription factors. Activated STAT dimers move towards the nucleus, binding to the special DNA sequences at the promotor region of the relevant gene that is responsive to the cytokine, and activate gene transcription. ${ }^{[16,17]}$

\section{JAK INHIBITORS}

\section{Tofacitinib}

Tofacitinib is an orally available, small-molecule JAK-family inhibitor of JAK1, JAK2, JAK3, and TYK2 that competitively binds to the active region of adenosine triphosphate kinase and prevents activation of signal transduction. ${ }^{[17,18]}$

\section{Baricitinib}

Another JAK inhibitor, Baricitinib, received approval for RA treatment in February 2017 and mainly shows minimal binding to JAK1, JAK2, as well as JAK3. Therefore, it inhibits many cytokines by blocking the signal through the $\gamma_{c}$ chain, $\beta$ common chain (IL-3, IL-5, and GM-CSF), gp130 (IL-6 family), interferons, and interleukins (IL-12, IL-23, IL-27). ${ }^{[19]}$

\section{Ruxolitinib}

Ruxolitinib (INCB018424) is a strong, selective JAK1 and JAK2 inhibitor. Its main effect is to prevent cell division and induce apoptosis by inhibiting JAK from phosphorylating STAT. ${ }^{[20]}$ Ruxolitinib (INCB018424) is the first tyrosine kinase inhibitory agent to be used in myelofibrosis treatment. ${ }^{[21]}$

\section{Peficitinib}

Peficitinib is an oral JAK1, JAK2, and JAK3 inhibitor that has 6-7 times greater in vitro potential for JAK3 compared to JAK1 and JAK2. ${ }^{[22]}$ 

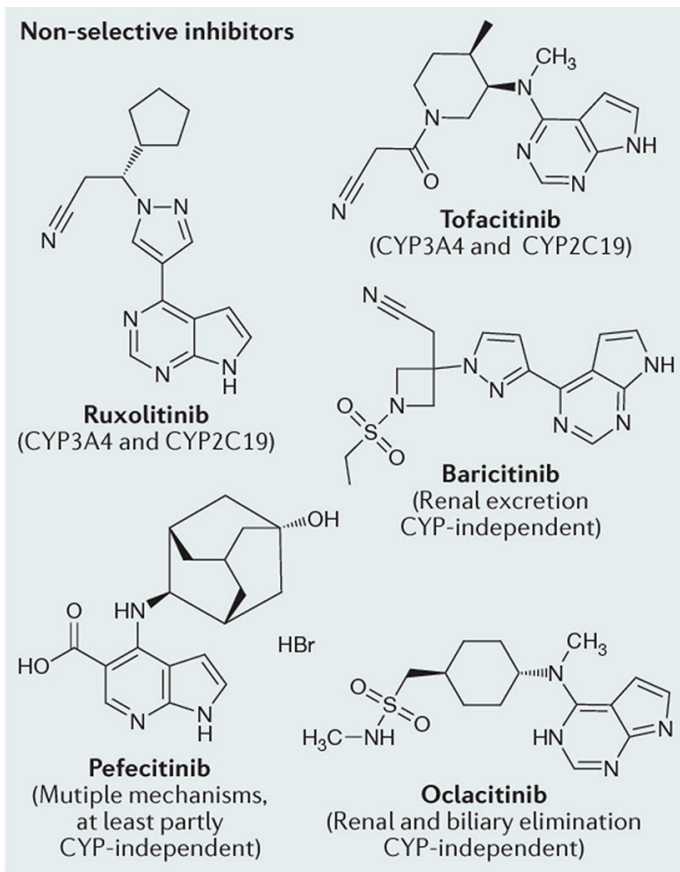

Inhibitors of JAK and other kinases

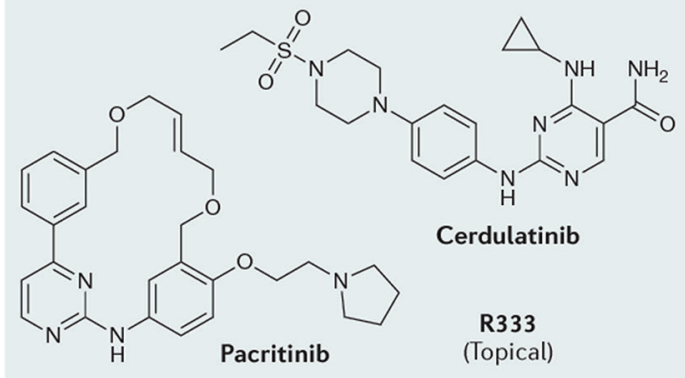

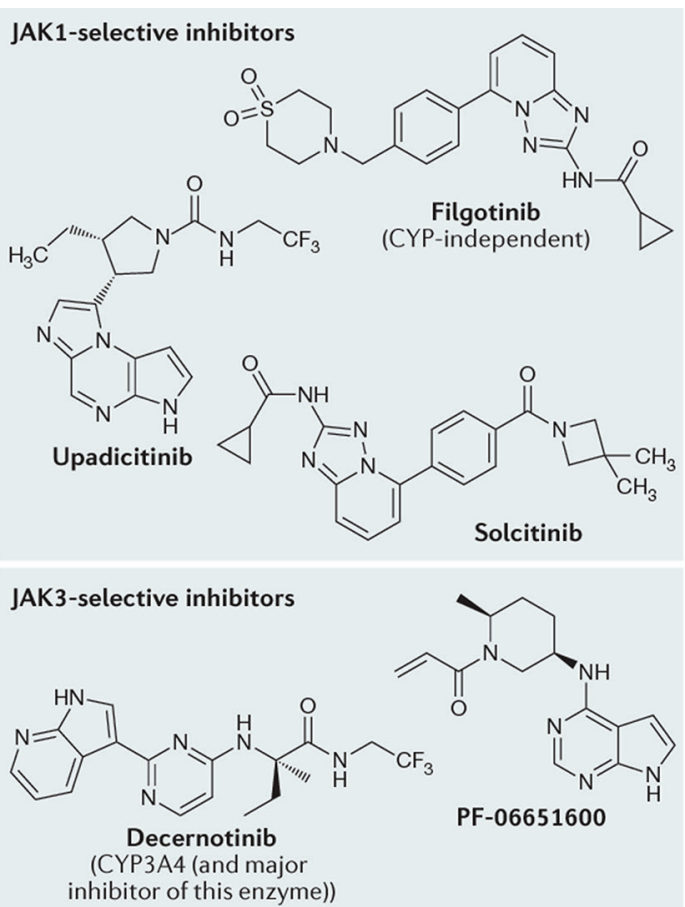

TYK2-selective inhibitors

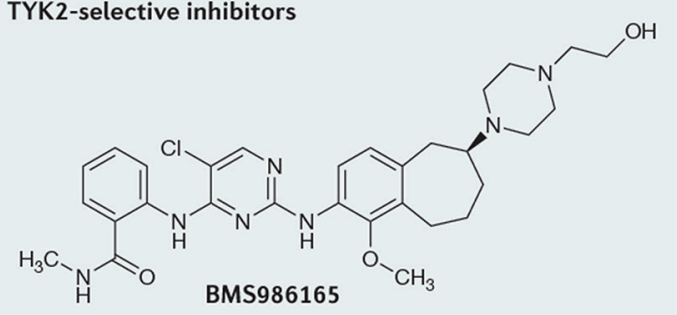

Also: NDI-031232, NDI-031407, PF-06700841 and SAR-20347

Figure 2. Types of janus kinase inhibitors and their chemical structures. ${ }^{[8]}$

\section{Upadacitinib}

Upadacitinib is a JAK1 inhibitor that was developed for the treatment of rheumatoid arthritis and other inflammatory diseases. Oral upadacitinib treatment is currently under study ${ }^{[23]}$ but so far there are no conclusive results. ${ }^{[24]}$

\section{Filgotinib}

Filgotinib was the first JAK1 inhibitor and may provide limited toxicity. It is well tolerated and has no effect on JAK2 signaling. ${ }^{.25]}$

\section{Momelotinib}

Momelotinib is a selective JAK1 and JAK2 inhibitor that may be beneficial in improving anemia in myelofibrosis patients. ${ }^{[26]}$

\section{Oclacitinib}

Oclacitinib is a new JAK inhibitor that targets cytokine activity involved in dermatologic dog allergies. Oclacitinib is most effective in inhibiting JAK1. ${ }^{[27]}$

\section{Decernotinib}

Decernotinib is a relatively new JAK inhibitor which has roughly five times more selectivity over JAK3 compared to other JAKs (JAK1, JAK2, TYK2) based on in vitro kinase assays. ${ }^{[26]}$ Studies on decernotinib have also shown an adverse reaction of lymphopenia, also known in tofacitinib. This could be associated with effects on JAK3 related cytokines such as IL-7 and IL-15. ${ }^{[28]}$ 


\section{Fedratinib}

Fedratinib is an orally available small molecule. It competes with the mutated form of AK2V617F as well as JAK2 for ATP binding, which may result in inhibition of JAK2 activity, JAK-STAT signaling pathway inhibition, and induction of tumor cell apoptosis. Its use was approved by the FDA on August 16, 2019. ${ }^{[29]}$

\section{Pacritinib}

Pacritinib is an orally available inhibitor of the JAK2 mutant JAK2V617F that has potential antineoplastic activity. ${ }^{[30]}$

\section{Cerdulatinib}

Cerdulatinib is a dual spleen tyrosine kinase (SYK) and JAK inhibitor which uniquely inhibits two key cell signaling pathways in certain hematological malignancies and autoimmune diseases. Both targets have been strongly shown to inhibit both SYK (B cell receptor pathway) and JAK (cytokine receptors) targets in $\mathrm{B}$ cell malignancies that have been shown to increase cancer cell growth and survival. ${ }^{[31]}$

\section{Solcitinib}

Solcitinib is a selective JAK1 inhibitor initially developed to treat systemic lupus erythematosus, psoriasis, and ulcerative chollitis. ${ }^{[32]}$

\section{Diseases in which inhibitors are used}

Skin diseases

Psoriasis, Lichen planus

Cutaneous lupus erythematosus

Pyoderma gangrenosum, atopic dermatitis

Alopecia areata, vitiligo, plaque psoriasis

Other diseases

Acute and chronic graft versus host disease,

Myeloproliferative neoplasms (MPN)

Rheumatoid arthritis (RA)

Severe combined immunodeficiency (SCID)

Ankylosing spondylitis (Low Back Rheumatism)

Juvenile idiopathic arthritis (Childhood Rheumatism)

\section{Declaration of conflicting interests}

The authors declared no conflicts of interest with respect to the authorship and/or publication of this article.

\section{Funding}

The authors received no financial support for the research and/or authorship of this article.

\section{REFERENCES}

1. Bittorf T, Seiler J, Lüdtke B, Büchse $T$, Jaster R, Brock J. Activation of STAT5 during EPO-directed suppression of apoptosis. Cell Signal 2000;12:23-30.

2. Jatiani SS, Baker SJ, Silverman LR, Reddy EP. Jak/STAT pathways in cytokine signaling and myeloproliferative disorders: approaches for targeted therapies. Genes Cancer 2010;1:979-93.

3. Thomas SJ, Snowden JA, Zeidler MP, Danson SJ. The role of JAK/STAT signalling in the pathogenesis, prognosis and treatment of solid tumours. Br J Cancer 2015;113:365-71.

4. Irino $\mathrm{T}$, Uemura $\mathrm{M}$, Yamane $\mathrm{H}$, Umemura $\mathrm{S}$, Utsumi T, Kakazu N. JAK2 V617F-Dependent Upregulation of PU.1 Expression in the Peripheral Blood of Myeloproliferative Neoplasm Patients Plos One 2011;6:1-10.

5. Santos FP, Verstovsek S. JAK2 inhibitors: are they the solution? Clin Lymphoma Myeloma Leuk 2011;11:2836.

6. Seavey MM, Dobrzanski P. The many faces of Janus kinase. Biochem Pharmacol 2012;83:1136-45.

7. Shuai K, Liu B. Regulation of JAK-STAT signalling in the immune system. Nat Rev Immunol 2003;3:900-11.

8. Schwartz DM, Kanno Y, O'Shea JJ. JAK inhibition as a therapeutic strategy for immune and inflammatory diseases. Published in Nature Reviews Drug Discovery 2017. Available at: https://www.semanticscholar.org/ paper/JAK-inhibition-as-a-therapeutic-strategy-forimmune-Schwartz-Kanno/29a7a6b1526753b1d0d37 0285c76432ae4675b0a

9. Ghoreschi K, Laurence A, O'Shea JJ. Janus kinases in immune cell signaling. Immunol Rev 2009;228:273-87.

10. Zou H, Yan D, Mohi G. Differential biological activity of disease-associated JAK2 mutants. FEBS Lett 2011;585:1007-13.

11. Welsch K, Holstein J, Laurence A, Ghoreschi K. Targeting JAK/STAT signalling in inflammatory skin diseases with small molecule inhibitors. Eur J Immunol 2017;47:1096-107.

12. Papp KA, Menter MA, Abe M, Elewski B, Feldman $\mathrm{SR}$, Gottlieb AB, et al. Tofacitinib, an oral Janus kinase inhibitor, for the treatment of chronic plaque psoriasis: results from two randomized, placebo-controlled, phase III trials. Br J Dermatol 2015;173:949-61.

13. Baxter EJ, Scott LM, Campbell PJ, East C, Fourouclas $\mathrm{N}$, Swanton S, et al. Acquired mutation of the tyrosine kinase JAK2 in human myeloproliferative disorders. Lancet 2005;365:1054-61.

14. Jones AV, Kreil S, Zoi K, Waghorn K, Curtis C, Zhang $\mathrm{L}$, et al. Widespread occurrence of the JAK2 V617F 
mutation in chronic myeloproliferative disorders. Blood 2005;106:2162-8.

15. Benekli M, Baer MR, Baumann H, Wetzler M. Signal transducer and activator of transcription proteins in leukemias. Blood 2003;101:2940-54.

16. Levy DE, Darnell JE Jr. Stats: transcriptional control and biological impact. Nat Rev Mol Cell Biol 2002;3:651-62.

17. Papp KA, Menter MA, Abe M, Elewski B, Feldman SR, Gottlieb AB, et al. Tofacitinib, an oral Janus kinase inhibitor, for the treatment of chronic plaque psoriasis: results from two randomized, placebocontrolled, phase III trials. 2015;173:949-61.

18. Riese RJ, Krishnaswami S, Kremer J. Inhibition of JAK kinases in patients with rheumatoid arthritis: scientific rationale and clinical outcomes. Best Pract Res Clin Rheumatol 2010;24:513-26.

19. Dougados M, van der Heijde D, Chen YC, Greenwald M, Drescher E, Liu J, et al. Baricitinib in patients with inadequate response or intolerance to conventional synthetic DMARDs: results from the RA-BUILD study. Ann Rheum Dis 2017;76:88-95.

20. Ostojic A, Vrhovac R, Verstovsek S. Ruxolitinib: a new JAK1/2 inhibitor that offers promising options for treatment of myelofibrosis. Future Oncol 2011;7:1035-43.

21. Yang LP, Keating GM. Ruxolitinib: in the treatment of myelofibrosis. Drugs 2012;72:2117-27.

22. Roskoski R Jr. Janus kinase (JAK) inhibitors in the treatment of inflammatory and neoplastic diseases. Pharmacol Res 2016;111:784-803.

23. A Study to Evaluate the Safety and Efficacy of Upadacitinib (ABT-494) for Induction and Maintenance Therapy in Subjects With Moderately to Severely Active Ulcerative Colitis (UC). NCT02819635. Available at: https://clinicaltrials.gov/ct2/show/NCT02819635

24. A Study to Evaluate the Long-Term Safety and
Efficacy of Upadacitinib (ABT-494) in Subjects With Ulcerative Colitis (UC). ClinicalTrials.gov Identifier: NCT03006068 Available at: https://clinicaltrials.gov/ ct2/show/NCT03006068

25. Vanhoutte F, Mazur M, Van der Aa A, Wigerinck P, van 't Klooster G. Selective JAK1 inhibition in the treatment of rheumatoid arthritis: Proof of concept with GLPG0634. Arthritis Rheum 2012;64:(Suppl 10):2489.

26. Astor L. FDA grants fast track designation to momelotinib for intermediate/high-risk myelofibrosis. Available at: https://www.targetedonc.com/news/ fda-grants-fast-track-designation-to-momelotinib-forintermediatehighrisk-myelofibrosis

27. Gonzales AJ, Bowman JW, Fici GJ, Zhang M, Mann

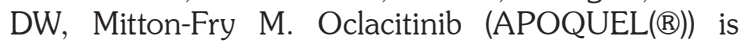
a novel Janus kinase inhibitor with activity against cytokines involved in allergy. $J$ Vet Pharmacol Ther 2014;37:317-24.

28. Mahajan S, Hogan JK, Shlyakhter D, Oh L, Salituro FG, Farmer L, et al. VX-509 (decernotinib) is a potent and selective janus kinase 3 inhibitor that attenuates inflammation in animal models of autoimmune disease. J Pharmacol Exp Ther 2015;353:405-14.

29. Macchi P, Villa A, Giliani S, Sacco MG, Frattini A, Porta F, et al. Mutations of Jak-3 gene in patients with autosomal severe combined immune deficiency (SCID). Nature 1995;377:65-8.

30. Available at: https://pubchem.ncbi.nlm.nih.gov/ compound/Fedratinib

31. Available at: https://pubchem.ncbi.nlm.nih.gov/ compound/Pacritinib

32. Blunt MD, Koehrer S, Dobson RC, Larrayoz M, Wilmore S, Hayman A, et al. The Dual Syk/JAK Inhibitor Cerdulatinib Antagonizes B-cell Receptor and Microenvironmental Signaling in Chronic Lymphocytic Leukemia. Clin Cancer Res 2017;23:2313-24. 